\title{
Modeling of nonlinear loads in high-voltage network by measured parameters
}

\author{
L.I. Kovernikova ${ }^{1}$, Luong Van Chung $^{2}$ \\ ${ }^{1}$ The Siberia Branch of the Russian Academy of Sciences \\ Energy Systems Institute, 130, Lermontov Str., 664033, Irkutsk (Russia) \\ Phone: +7 3952500646 ext. 232, fax: +7 3952 426796, e-mail: kovernikova@isem.irk.ru \\ ${ }^{2}$ National Research Irkutsk State Technical University \\ Hung Yen (Vietnam) \\ e-mail: chunglv@mail.ru
}

\begin{abstract}
The paper presents an algorithm for modeling nonlinear loads connected to the nodes of high-voltage network. The algorithm is developed on the basis of measured parameters of harmonic conditions. The high-voltage networks are extended. Many nonlinear loads distributed across the territory of the region are connected to them. An analysis of measured parameters of harmonic conditions shows that they vary randomly. The algorithm for modeling the nonlinear loads is developed considering their probabilistic properties. The algorithm is illustrated with an example of modeling the nonlinear loads of a railway traction substation and an aluminum smelter shop that are powered by a $220 \mathrm{kV}$ network.
\end{abstract}

\section{Key words}

Harmonics, high-voltage network, measurement, statistical analysis, nonlinear load model

\section{Introduction}

Publications propose many ideas on modeling nonlinear loads. Some general principles of modeling are presented in [1], [2]. Models of various electric equipment with nonlinear voltage-current characteristics are presented in [3], [4]. In [5] authors propose a technique for modeling an aggregate nonlinear load on the basis of measurements for distribution networks supplying power to consumers of industrial, commercial and residential sectors. Modeling of nonlinear loads connected to the nodes of high-voltage $(110-220 \mathrm{kV})$ network is an unsolved problem. The parameters of harmonic conditions are largely determined by specific features of high-voltage networks.

The high-voltage networks are extended. They are spread over a large territory. Many high-power nonlinear loads are connected to the nodes of high-voltage networks. Each of the loads represents a facility that has its own electric network of a lower voltage. The network of the facility supplies power to different items of equipment including that with nonlinear voltage-current characteristics. An analysis of high-voltage network conditions cannot take into account and represent each items of equipment of the facility in the calculation scheme. The main technological electrical equipment of the facility is the source of harmonics. Currents of these harmonics are drawn from the high-voltage network to the medium- and low-voltage networks. High-voltage networks supply power to medium- and low-voltage networks. They contain also a great amount of electrical equipment with nonlinear voltage-current characteristics. Harmonic currents from these networks are drawn to the high-voltage networks. Thus, the medium- and low-voltage networks can be considered as a nonlinear load connected to the highvoltage network node.

Taking into account the above specific features we can state that measurements are the only way to receive information on a range of harmonic currents, their magnitudes and phases for modeling the nonlinear loads connected to the nodes of high-voltage networks. Duration of measurements should be determined individually for each certain case. Since the parameters of harmonic conditions in high-voltage networks vary randomly [6]-[8] it is necessary to analyze them by the methods of mathematical statistics.

In this paper we analyze the measured parameters of harmonic conditions on the basis of data measured at connection nodes of a railway traction substation and an aluminum smelter shop. Based on the obtained results, we developed an algorithm to model the nonlinear loads.

\section{Mathematical statement of the problem}

The parameters of harmonic conditions are calculated by solving a system of equations for each harmonic [9]

$$
U_{h}=Z_{h} I_{h},
$$

where $h$-harmonic order, $\boldsymbol{U}_{\boldsymbol{h}}$ - column-matrix of nodal voltage values to be determined, $\boldsymbol{Z}_{\boldsymbol{h}}$ - a square matrix of self- and mutual impedances of network nodes, $\boldsymbol{I}_{\boldsymbol{h}}$ column-matrix of harmonic currents at network nodes 
representing load. Each element $i$ of matrix $\boldsymbol{I}_{\boldsymbol{h}}$ is a complex number $I_{i h}=I a_{i h}+j I r_{i h}$.

The objective is to determine current $\boldsymbol{I}_{\boldsymbol{h}}$ by the measurement results. If network has one load with nonlinear voltage-current characteristic there will be no problems in determining current $\boldsymbol{I}_{\boldsymbol{h}}$. Whereas if the network has several nonlinear loads connected to different nodes then their harmonic currents are spread over the network and penetrate into the loads of virtually all nodes. In this case they are random values. Their random nature is determined by varying topology of the network, equipment, wave and frequency properties of the network, phases of harmonic source currents, voltage at nonlinear equipment, etc.

An equivalent circuit of high-voltage network with a nonlinear load connected to its node is traditional and it is presented in Fig.1.

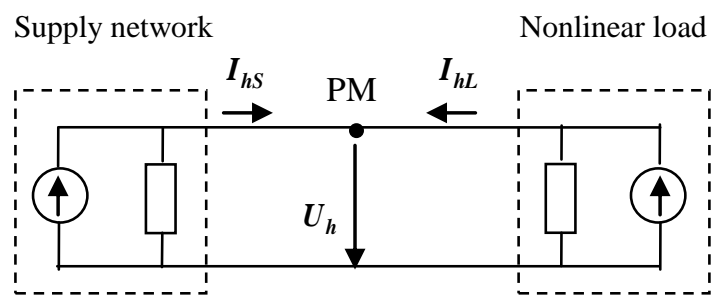

Fig. 1. An equivalent circuit of a supply network and a nonlinear load for the $h$-th harmonic.

The notations used in the scheme are:

$I_{h S}$ - the $h$-th harmonic current phasor of the supply network,

$\boldsymbol{I}_{h L}$ - the $h$-th harmonic current phasor of nonlinear load,

$\mathrm{PM}$ - the point of measurement.

Current $\boldsymbol{I}_{h S}$ is a resultant current of all nonlinear loads available in the network except for current $\boldsymbol{I}_{h L}$. In Fig. 1, the harmonic current $\boldsymbol{I}_{\boldsymbol{h}}$ drawn through the point of measurement is determined by the vector sum of currents $I_{h S}$ and $I_{h L}$, i.e.

$$
I_{h}=I_{h S}+I_{h L} .
$$

Based on the measurements at the connection node we obtained the effective values and phase angles $\left(I_{h}, \varphi_{I h}, \varphi_{U h}\right)$ of current $\boldsymbol{I}_{\boldsymbol{h}}$ and voltage $\boldsymbol{U}_{\boldsymbol{h}}$. The measurements were performed during 24 hours with a time interval of 1 minute. As a result, we obtained an array of 1440 elements. Angles $\varphi_{I h}$ and $\varphi_{U h}$ make it possible to determine the phase angle $\varphi_{h}$ between current and voltage of the $h$-th harmonic by

$$
\varphi_{h}=\varphi_{U h}-\varphi_{I h} \text {. }
$$

In [10] angle $\varphi_{h}$ determines the directions of active and reactive power flows with respect to the point of measurement. The angle can also be applied to determine the directions of active and reactive components of harmonic currents. The values of active $\left(I_{a h}\right)$ and reactive $\left(I_{r h}\right)$ currents are calculated according to [11], using

$$
I_{a h}=I_{h} \cos \varphi_{I h}, I_{r h}=I_{h} \sin \varphi_{I h} .
$$

An analysis of directions of active and reactive currents allows us to make a conclusion whether the node is a generator node or a load node for a certain harmonic in order to represent appropriately the model of current $\boldsymbol{I}_{\boldsymbol{h}}$ in the system of equations (1).

The previous analysis of measured data in [2], [6]-[8] shows that modeling a nonlinear load should include two stages:

1) analysis of directions of active and reactive harmonic currents,

2) development of models of active and reactive harmonic currents based on the results of the first stage.

\section{Analysis of directions of active and reactive harmonic currents}

According to [10] the direction from network to load is assumed to be a positive direction of active current and active power. For reactive current we assume the same direction as for reactive power in [11], provided the load is inductive. Active and reactive currents are directed from network to load if angle $\varphi_{h}$ lies in the interval from 0 to $\pi / 2$, i.e. in the first quadrant of a complex plane. Active current is directed from load to network and reactive from network to load, if angle $\varphi_{h}$ lies in the interval from $\pi / 2$ to $\pi$, i.e. in the second quadrant. Both currents are directed from load to network if angle $\varphi_{h}$ lies in the interval from $\pi$ to $3 \pi / 2$, i.e. in the third quadrant. Active current is directed from network to load and reactive - in an opposite direction, if angle $\varphi_{h}$ lies in the interval from $3 \pi / 2$ to $2 \pi$, i.e. in the fourth quadrant. Thus, in each quadrant there is a certain direction of active and reactive harmonic currents.

In [8] based on the analysis of measurements we obtained that at one and the same time instant active power of different harmonics can have opposite directions. This conclusion applies to currents as well. The analysis of directions of active and reactive currents was made for one phase of a railway traction substation and an aluminum smelter shop for harmonics 3, 5, 7, 9, 11, 13, 23,25 . The results of the analysis are presented in Table I. Notations of the table are: RTS - railway traction substation. ASS - aluminum smelter shop.

Table I. - Distribution of angles $\varphi_{h}(\%)$

\begin{tabular}{|c|c|c|c|c|c|c|c|c|}
\hline \multirow{3}{*}{$h$} & \multicolumn{4}{|c|}{ RTS } & \multicolumn{4}{c|}{ ASS } \\
\cline { 2 - 9 } & \multicolumn{3}{|c|}{ Quadrants } & \multicolumn{4}{c|}{ Quadrants } \\
\cline { 2 - 9 } & 1 & 2 & 3 & 4 & 1 & 2 & 3 & 4 \\
\hline 3 & 10.3 & 14.7 & $\mathbf{3 7 . 2}$ & $\mathbf{3 7 . 8}$ & 22.2 & $\mathbf{6 0 . 6}$ & 11.2 & 6.0 \\
\hline 5 & $\mathbf{8 1 . 7}$ & 5.1 & 0.3 & 12.9 & 20.7 & 7.4 & $\mathbf{3 6 . 8}$ & $\mathbf{3 5 . 1}$ \\
\hline 7 & 0.8 & 19.5 & $\mathbf{6 8 . 5}$ & 11.1 & 0.2 & 0.0 & 22.4 & $\mathbf{7 7 . 4}$ \\
\hline 9 & $\mathbf{2 6 . 2}$ & 22.4 & 21.9 & $\mathbf{2 9 . 5}$ & 7.1 & 30.8 & $\mathbf{5 3 . 8}$ & 8.2 \\
\hline 11 & 21.7 & $\mathbf{3 2 . 1}$ & $\mathbf{2 9 . 4}$ & 16.8 & $\mathbf{7 4 . 4}$ & 7.0 & 0.1 & 18.5 \\
\hline 13 & 19.5 & $\mathbf{2 6 . 9}$ & $\mathbf{3 3 . 7}$ & 19.9 & 6.5 & $\mathbf{4 4 . 5}$ & $\mathbf{2 9 . 7}$ & 19.2 \\
\hline 23 & $\mathbf{6 0 . 3}$ & $\mathbf{2 6 . 8}$ & 4.5 & 8.4 & $\mathbf{5 5 . 5}$ & 16.7 & 5.6 & 22.3 \\
\hline 25 & $\mathbf{4 5 . 7}$ & 23.0 & 11.0 & 20.3 & $\mathbf{4 5 . 9}$ & $\mathbf{3 7 . 5}$ & 9.2 & 7.4 \\
\hline
\end{tabular}


The Table presents the number of measurements in percentage of the total number of 1440 measurements that correspond to the distributions of directions of active and reactive currents over the quadrants of the complex plane. The data presented in the Table show that the distribution of current directions across the quadrants is different. Some harmonic currents have predominant directions, i.e. above $50 \%$ of measurement time. This applies to the currents of harmonics 5, 7 and 23 of the railway traction substation and currents of harmonics $3,7,9,11$, and 23 of the aluminum smelter shop. The harmonic processes are of random character and even insignificant changes in the operating conditions of the network can affect them. The predominant directions will not change since they are determined by the load conditions and network configuration. At harmonic 25 of the railway traction substation and harmonics 13 and 25 of the aluminum smelter shop there are the most pronounced directions although they make up less than 50\%. At these harmonics there is one more direction for which the quantity of measurements considerably exceeds the remaining two. In such cases it is suggested to develop the models of loads for two variants. Harmonics 3, 9, 11 and 13 of the railway traction substation and harmonic 5 of the aluminum smelter shop have two directions that prevail but their quantities differ little from one another. In such cases it is necessary to take two variants to develop a model. Based on the analysis of Table I, to model harmonic current we assume the variants highlighted in bold.

\section{An algorithm to model a current of one harmonic}

Measured parameters $I_{h}, \varphi_{I h}, \varphi_{U h}$ represent time series of random discrete values. Solving the system of equations (1) requires the values of active and reactive currents with a probability of 0.95 . The values of parameters with a probability of 0.95 are used in the standard [12] in the assessment of voltage quality. To calculate currents with a probability of 0.95 it is necessary to know the distribution functions $\left(f(x)\right.$, where $x=I_{a h}$ or $\left.x=I_{r h}\right)$. The distribution function of a random value is determined on the basis of a probability density function. Thus, the aim of the algorithm is to identify the probability density functions of active and reactive harmonic currents, and then calculate the values of currents with a probability of 0.95 , using respective distribution functions.

Figure 2 presents a block-diagram of an algorithm for processing a time series of random values of active and reactive currents of one harmonic. The algorithm is based on an analysis of special literature, including [13]-[15]. Below the most important points of the algorithm are described in detail. Construction of a scatter plot (Step 1 in Fig.1) of the measured time series of random values makes it possible to visually determine the presence of abnormal elements whose values considerably exceed the values of the remaining elements. In [15] the abnormal elements are called outliers. They are well seen in the scatter plot. The outliers can be replaced with neighboring elements, a mean value of neighboring elements or by other ways proposed in special literature [13], [14].

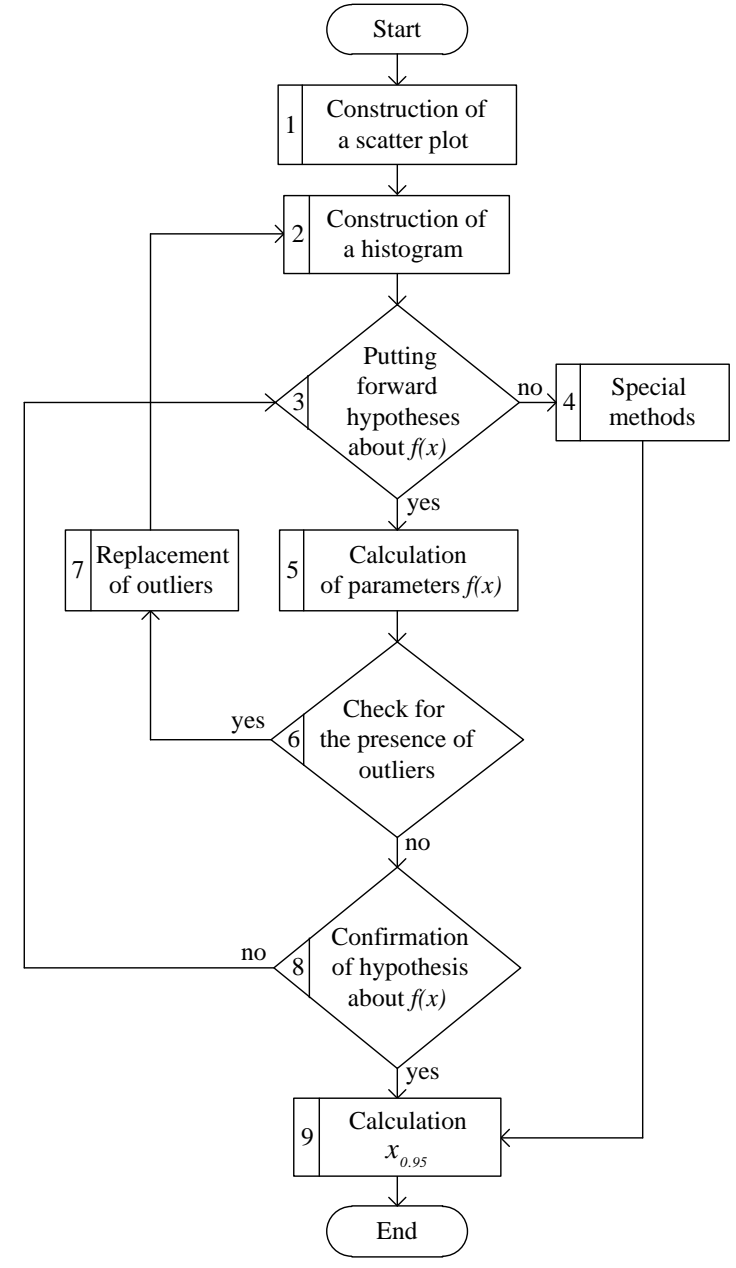

Fig. 2. A block-diagram of an algorithm for modeling a harmonic current.

The construction of a histogram and visual analysis of its shape (Step 2) make it possible to put forward the hypotheses on the identification of probability density function of current. In the event that it is impossible to identify the probability density function by the histogram shape, then special approaches should be used (Step 4). Some approaches are presented in [2], [13], [14]. If the hypotheses on the probability density function are put forward, then each hypothesis should be tested further by the algorithm.

First of all, it is necessary to calculate the parameters (Step 5) for an analytical description of the probability density function according to [13], [14], by using an array of measured random values of current. Then, by using special criteria developed for specific functions [13]-[15], check if there are large outliers and very small outliers (Step 6). In the event that there are outliers they should be replaced as was indicated above. Then the processing procedure is repeated starting with Step 2 since the replacement of series elements can change the histogram shape. In the event that there are no outliers, the hypothesis about the probability density function is checked (Step 8), for example, by the goodness-of-fit tests of Pearson, Kolmogorov-Smirnov [13], [14]. In the case 
that the first hypothesis is not confirmed, it is necessary to test the second hypothesis starting with Step 3. If none of the put forward hypotheses is confirmed by the tests, it is necessary to apply special methods (Step 4). In the case that one of the hypotheses about the probability density function is confirmed then the distribution function is used to calculate the value of current with a probability of 0.95 . The calculation of the current value with a set probability completes the modeling of harmonic current of nonlinear load.

\section{A case study on the algorithm application}

The operation of the algorithm is illustrated by modeling the active current of the 5-th harmonic of the railway traction substation.

Step 1. We construct a scatter plot of the current (Fig.3). The visual analysis shows that there are no large outliers.

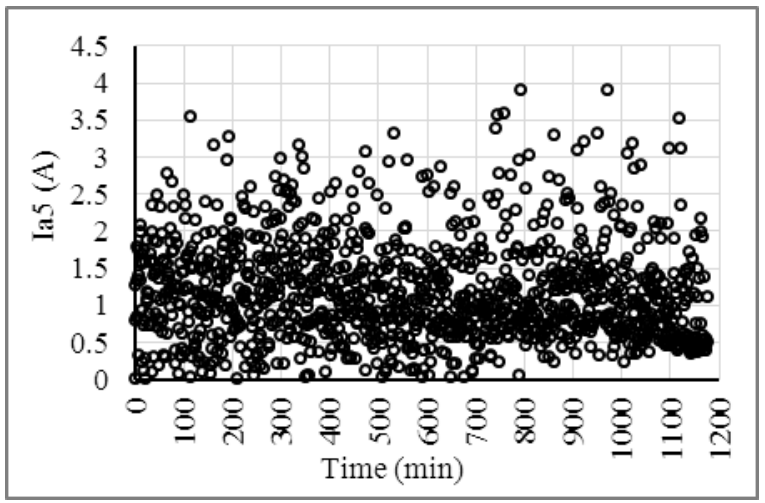

Fig. 3. Scatter plot of active current $I_{a 5}$.

Step 2. We construct a histogram (Fig.4), make a visual analysis to identify the probability density function.

Step 3. We put forward hypotheses about the probability density functions. Based on the analysis of the histogram shape two hypotheses are put forward. The first hypothesis is that the histogram shape is close to the Rayleigh probability density function, i.e.

$$
f(x, a)=\frac{x}{a^{2}} \exp \left(-\frac{x^{2}}{2 a^{2}}\right), \text { where } x \geq 0 .
$$

The second hypothesis is that the histogram shape is close to the Weibull probability density function, i.e.

$$
f(x, \alpha, \beta)=\frac{\beta}{\alpha^{\beta}} x^{\beta-1} \exp \left[-\left(\frac{x}{\alpha}\right)^{\beta}\right], \quad \text { where } x \geq 0, \quad \alpha>0,
$$$$
\beta>0 \text {. }
$$

Step 4 is skipped.

Step 5. For an analytical description of the Rayleigh function we should calculate one parameter $a$. It is calculated by an expression from [13]. Its value equals 0.94 .

Step 6. To check if there are outliers in the array of random values which are described by the Rayleigh distribution function $F(x, a)=1-\exp \left(-\frac{x^{2}}{2 * 0.94^{2}}\right) \quad$ we apply the Darling test [13]. The calculations show that there are no outliers.

Step 7 is skipped.

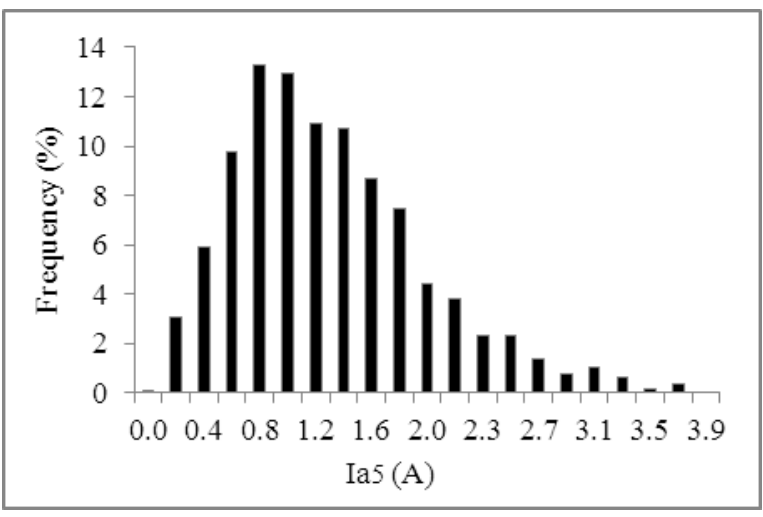

Fig. 4. Histogram of current $I_{a 5}$.

Step 8. To confirm the correspondence between the histogram shape and the Rayleigh density function we use the Pearson goodness-of-fit test [13]. After the necessary calculations are carried out, we obtain an experimental value of criterion $\chi_{э \kappa}^{2}$ equal to 67.64. Critical value $\chi_{\kappa p}^{2}$ at a significance level of 0.05 and the number of degrees of freedom 18 is equal to 28.87. Since $\chi_{э \kappa}^{2}>\chi_{\kappa p}^{2}$, the hypothesis about the Rayleigh probability density function is not confirmed. We go back to Step 3.

Step 3. We test the second hypothesis about the Weibull density function.

Step 4 is skipped.

Step 5. For an analytical description of the Weibull density function it is necessary to calculate two parameters $\alpha$ and $\beta$. They are calculated by the expressions from [13]. The values $\alpha$ and $\beta$ appeared to be equal to 1.32 , and 1.81 .

Step 6. To check if there are outliers in the array of random values which are defined by the Weibull distribution function $F(x, \alpha, \beta)=1-\exp \left[-\left(\frac{x}{1.32}\right)^{1.81}\right]$ we apply the Darling test. The calculations show that there are no outliers.

Step 7 is skipped.

Step 8. To confirm the correspondence between the histogram shape and the Weibull density function we apply the Pearson goodness-of-fit test. After the calculations we obtain that the experimental value of criterion $\chi_{э \kappa}^{2}$ equals 24.77 . The critical value $\chi_{\kappa p}^{2}$ at a significance level of 0.05 and the number of degrees of freedom 18 equals 28.87 . Since $\chi_{\ni \kappa}^{2}<\chi_{\kappa p}^{2}$, the hypothesis about the Weibull probability density function is confirmed.

Step 9. To determine the value of current with a probability of 0.95 we use the Weibull distribution function that corresponds to the calculated parameters $\alpha$ and $\beta$, i.e. $0.95=1-\exp \left[-\left(\frac{x_{0.95}}{1.32}\right)^{1.81}\right]$. By solving the last equality we have $x_{0.95}=2.42$. The active component of current with a probability of 0.95 will not exceed $2.42 \mathrm{~A}$. The reactive component of current, obtained by the described algorithm with a probability of 0.95 , will not exceed 3.05A. Thus, we obtain the 5-th harmonic current 
equal to $I_{5}=2.42+j 3.05 \mathrm{~A}$ for solving the system of equations (1). Table II presents the probability density functions and the values of active and reactive harmonic currents for the variants highlighted in bold in Table I.

Table II. - Probability density functions of harmonic currents

\begin{tabular}{|c|c|c|c|c|}
\hline \multirow{2}{*}{$h$} & \multicolumn{2}{|c|}{ RTS } & \multicolumn{2}{|c|}{ ASS } \\
\hline & $I a$ & $I r$ & $I a$ & $I r$ \\
\hline \multirow{4}{*}{3} & Exp & Exp & \multirow{2}{*}{ Special } & \multirow{2}{*}{ Beta } \\
\hline & 3.9 & 4.8 & & \\
\hline & Exp & Gamma & 22 & 26 \\
\hline & 4.2 & 4.7 & 2.2 & 2.0 \\
\hline \multirow{4}{*}{5} & \multirow{2}{*}{ Weibull } & \multirow{2}{*}{ Weibull } & Weibull & Gauss \\
\hline & & & 3.83 & 4.0 \\
\hline & \multirow{2}{*}{2.4} & \multirow{2}{*}{3.1} & Special & Weibull \\
\hline & & & 3.8 & 4.2 \\
\hline \multirow{2}{*}{7} & Beta & Beta & Beta & Gauss \\
\hline & 1.4 & 1.6 & 2.5 & 3.6 \\
\hline \multirow{4}{*}{9} & Exp & Exp & \multirow{2}{*}{ Rayleigh } & \multirow{2}{*}{ Weibull } \\
\hline & 0.8 & 0.7 & & \\
\hline & Beta & Beta & \multirow{2}{*}{1.0} & \multirow{2}{*}{0.9} \\
\hline & 0.7 & 0.7 & & \\
\hline \multirow{4}{*}{11} & Exp & Exp & \multirow{2}{*}{ Special } & \multirow{2}{*}{ Beta } \\
\hline & 0.7 & 0.7 & & \\
\hline & Weibull & Beta & \multirow{2}{*}{22.5} & \multirow{2}{*}{22.5} \\
\hline & 0.5 & 0.5 & & \\
\hline \multirow{4}{*}{13} & Beta & Exp & Special & Special \\
\hline & 0.5 & 0.5 & 17.3 & 15.3 \\
\hline & Beta & Beta & Special & Special \\
\hline & 0.5 & 0.5 & 18.1 & 18.7 \\
\hline \multirow{4}{*}{23} & Weibull & Weibull & \multirow{2}{*}{ Special } & \multirow{2}{*}{ Special } \\
\hline & 0.2 & 0.4 & & \\
\hline & Beta & Weibull & 35 & 26 \\
\hline & 0.1 & 0.3 & $5 . J$ & 2.0 \\
\hline \multirow{4}{*}{25} & \multirow{2}{*}{ Weibull } & \multirow{2}{*}{ Beta } & Beta & Extremal \\
\hline & & & 3.1 & 2.9 \\
\hline & \multirow{2}{*}{0.3} & 03 & Beta & Special \\
\hline & & & 3.3 & 3.2 \\
\hline
\end{tabular}

Notations of the table are: Exp - exponential, Weibull, Gauss, Rayleigh, Beta, Gamma, Extremal distribution functions, Special - special methods.

\section{Conclusion}

1) Based on the measured parameters of harmonic conditions at the nodes connecting nonlinear loads to the high-voltage network we proposed a methodological approach to modeling the nonlinear loads. The approach consists of two stages: an analysis of directions of active and reactive harmonic currents, and development of models of active and reactive harmonic currents, based on the analysis results.

2) We propose an algorithm to model the harmonic currents of nonlinear loads. The algorithm takes into account the probabilistic properties of the parameters of harmonic conditions.
3) The analysis of active and reactive currents of different harmonics of the railway traction substation and aluminum smelter shop shows that these currents are described by the distribution functions of Gauss, Rayleigh, Weibull, exponential, beta, extremal and gamma.

\section{References}

[1] IEEE Power Engineering Society. Tutorial on harmonics modeling and simulation. 1998.

[2] Probabilistic aspects task force of the harmonics working group subcommittee of the transmission and distribution committee, "Time-varying harmonics: part I - characterizing measured data", IEEE Trans. on Power Delivery, vol.13, No. 3, July 1998, pp. 938-943.

[3] A. Mansur, W.M. Grady, A.H. Chowdhury, M.J. Samotyj, An investigation of harmonics attenuation and diversity among distributed single-phase electronic loads, IEEE Transactions on Power Delivery, vol. 10, No. 1, January 1995, pp. 467-473.

[4] Luis Sainz, Juan Jose Mesas, Albert Ferrer, Characterization of non-linear load behavior, Electric Power systems Research 78 (2008), pp. 1773-1783.

[5] Mau Teng Au, Jovica V. Milanovic, Development of stochastic aggregate harmonic load model based on field measurements, IEEE Transactions on Power Delivery, vol. 22, No. 1, January 2007, pp. 323-330.

[6] L.I. Kovernikova, Results of the research into the harmonics of loads connected to the nodes of high- voltage network, Proceeding of International Conference on Renewable Energies and Power Quality (ICREPQ'14), Cordoba (Spain), 8th to 10th April, 2014.

[7] L.I. Kovernikova, Analysis of probabilistic properties of harmonic currents of loads connected to the high voltage networks, Proceeding of International conference on renewable energies and power quality (ICREPQ'15), La Coruña (Spain), 25th to 27th March, 2015.

[8] L.I. Kovernikova, Research into harmonic power in the highvoltage networks, Proceeding of International conference on renewable energies and power quality (ICREPQ'16, Madrid (Spain), 4th to 6th May, 2016.

[9] Arrillaga, Jos. Power system harmonics / J. Arrillaga, N.R. Watson. -2nd edit. - Chichester: Wiley, 2003.

[10] R.H. Stevens, Power flow direction definitions for metering bidirectional power, IEEE Transactions on Power Apparatus and Systems, Vol.102, No. 9, Sept. 1983, pp. 3018-3021.

[11] A.E. Emanuel, Power definitions and physical mechanism of power flow, John Wiley \&Sons, 2010.

[12] State standard 32144-2013. Power quality limits in public power supply systems. Moscow. Standartinform. 2014 (in Russian).

[13] Kobzar A.I. Applied mathematical statistics. For engineers and researchers. 2-nd edition, revised - M.: FIZMATLIT, 2012 (in Russian).

[14] B.Yu. Lemeshko, S.B. Lemeshko, S.N. Postovalov, E.V. Chimitova, Statistical data analysis, simulation and study of probability regularities. Computer approach. Novosibirsk : NSTU Publisher, 2011(in Russian).

[15] J.O. Irwin, On a criterion for the rejection of outlying observations. Biometrika, 1925, Volume 17, Issue 3-4. 